\title{
Development of a physics-based model to predict the performance of pumps as turbines
}

\author{
Mauro Venturini*, Lucrezia Manservigi, Stefano Alvisi, Silvio Simani \\ Università degli Studi di Ferrara, Ferrara, Italy
}

\section{A R T I C L E I N F O}

\section{Keywords:}

Pump as turbine

Pump

Performance curve

Simulation model

Hydraulic energy

Optimization

\begin{abstract}
A B S T R A C T
This paper presents the development of a physics-based simulation model, aimed at predicting the performance curves of pumps as turbines (PATs) based on the performance curves of the respective pump. The simulation model implements the equations to be used for the estimation of head, power and efficiency for both direct and reverse operation. Model tuning on a given machine is performed by using loss coefficients and specific parameters identified by means of an optimization procedure, which is first applied to the considered pumps and subsequently to the same machine running in PAT mode.

The simulation model is calibrated on data taken from literature, reporting both pump and PAT performance curves for head, power and efficiency over the entire range of operation. The performance data were acquired experimentally from four different centrifugal pumps, running in both pump and PAT mode and characterized by specific speed values in the range of 1.53-5.82. The accuracy of the predictions of the physics-based simulation model is quantitatively assessed against both pump and PAT experimental performance curves. Prediction consistency from a physical point of view is also evaluated.

The results presented in this paper highlight that all the performance curves predicted by the simulation model are physically consistent over the entire range of operation. In general, the prediction error on the head of PATs is acceptable, while the accuracy of the prediction of PAT power, and thus of PAT efficiency, is more case-sensitive and usually higher. The relative deviation of model prediction with respect to the field data regarding head and power at the PAT best efficiency point always seems acceptable compared to the uncertainty of the original experimental data and to typical deviations of other methods available in literature.

As a conclusion, the physics-based simulation model developed in this paper represents a powerful and reliable tool for estimating PAT performance curves over the entire range of operation based on pump characteristics.
\end{abstract}

\begin{tabular}{ll|} 
P & power \\
PAT & pump as turbine \\
Q & volume flow rate \\
RMSE & root mean square relative error \\
S & casing clearance \\
$u$ & circumferential velocity \\
X & parameter for pump/PAT model tuning \\
y & model parameter \\
Y & nondimensional performance parameter $(\pi, \eta, \psi)$ \\
Z & hydraulic loss \\
$\alpha$ & angle between direction of circumferential and absolute \\
& velocity \\
$\beta$ & angle between relative velocity vector and negative di- \\
& rection of circumferential velocity \\
$\varepsilon$ & wrap angle
\end{tabular}

\footnotetext{
* Corresponding author.

Email address: mauro.venturini@unife.it (M. Venturini)
} 


\begin{tabular}{|ll|}
\hline & \\
$\eta$ & loss coefficient \\
$\lambda$ & efficiency \\
$\pi$ & angle between vanes and side disks \\
$\rho$ & nondimensional power defined as $\mathrm{P} /\left(\mathrm{\rho n}^{3} \mathrm{D}^{5}\right)$ \\
$\phi$ & density \\
$\psi$ & nondimensional volume flow rate defined as $\mathrm{Q} /\left(\mathrm{nD}^{3}\right)$ \\
$\omega$ & nondimensional head defined as $\mathrm{gH} /\left(\mathrm{n}^{2} \mathrm{D}^{2}\right)$ \\
$\Omega$ & angular velocity \\
$\mathrm{A}$ & specific speed defined as $\omega \cdot \mathrm{Q}^{0.5} /(\mathrm{gH})^{0.75}$ \\
$\mathrm{ax}$ & outlet casing \\
$\mathrm{B}$ & blade \\
$\mathrm{BEP}$ & best efficiency point \\
$\mathrm{e}$ & experimental \\
$\mathrm{E}$ & inlet casing \\
$\mathrm{er}$ & friction created by the components of axial thrust bal- \\
& ance devices \\
$\mathrm{h}$ & hydraulic, hydrostatic bearing \\
int & calculated by means of interpolation curves \\
$\mathrm{k}$ & index of pump/PAT $(\mathrm{k}=1,2,3,4)$ \\
$\mathrm{La}$ & impeller \\
Le & diffuser \\
$\mathrm{m}$ & mechanical, meridional component \\
$\mathrm{P}$ & pump \\
$\mathrm{Rec}$ & recirculation \\
$\mathrm{RR}$ & disk friction \\
$\mathrm{S}$ & simulated \\
$\mathrm{s} 3$ & throttling \\
$\mathrm{sp}$ & volute \\
$\mathrm{st}$ & stage \\
$\mathrm{T}$ & PAT \\
th & theoretical \\
$\mathrm{u}$ & useful \\
$\mathrm{V}$ & volumetric \\
$\mathrm{Y}$ & nondimensional parameter \\
$\eta$ & efficiency \\
$\pi$ & nondimensional power \\
$\psi$ & nondimensional head \\
& \\
& \\
\hline & \\
\hline &
\end{tabular}

\section{Introduction}

Today, hydropower accounts for more than $16 \%$ of the world's net electricity production, according to a recent study carried out by Balkhair and Rahman [1].

Small hydro power plants are one of the most important renewable energy generation sources for developing countries. In fact, they represent a cost-effective technology that is being used for rural electrification in developing countries, such as India [2,3]. Small-scale hydropower systems are also becoming increasingly successful options for hydropower generation in small localities and remote areas, as demonstrated in [1] for a case study in Pakistan. Another example is provided by a small-scale hydro/PV/wind-based hybrid electric supply system located in Ethiopia and analyzed by Bekele and Tadesse in [4].

Micro-hydropower also presents new opportunities for generating electricity from the existing water infrastructure in OECD countries. One of the first examples of such an opportunity was presented by Bakos in [5] within the framework of a hybrid wind/hydropower system aimed at producing low-cost electricity in Greece and in [6] by Zakkour et al., where the recoverable power in the UK water industry was estimated in the order of $17 \mathrm{MW}$. More recently, Carravetta et al. [7] estimated the theoretical convertible power in the European Union area to be equal to 28.5 MW
Several papers have investigated the possibility of converting waste hydraulic energy in the water supply and distribution networks into useful electric energy. Giugni et al. analyzed different approaches suitable for locating and setting turbines in order to maximize their effectiveness and minimize water losses [8]. Puleo et al. developed a hydraulic model to evaluate the potential energy recovery from the use of centrifugal pumps as turbines (PATs) in a water distribution network characterized by the presence of private tanks [9]. Carravetta et al. investigated the benefit of a combination of a PAT, two regulating valves and two pressure meters in urban pipe networks [10]. Sitzenfrei et al. exploited water surplus in water distribution systems in order to maximize profits over one decade of operation [11]. Rossi et al. performed some laboratory tests to investigate pump performance in turbine mode in the case of an aqueduct installation [12]. Capelo et al. studied the application and optimization of a PAT in water systems when the type of recovery solution is off-grid [13]. Meirelles Lima et al. proposed a method for identifying the best network location for installing PATs [14]. Kramer et al. [15] presented the results of two research projects undertaken in collaboration with local drinking water supply companies. An extensive review of suitable hydraulic machinery, an evaluation of the energy recovery potential within the South German drinking water supply system and several field studies (including investment costs) were presented and discussed.

A structured four-step methodology for assessing potential energy recovery sites in water and wastewater infrastructures in regions of the UK and Ireland was presented by Gallagher et al. in [16]. The same authors also analyzed the potential for eco-design measures to improve the environmental and resource balance of five small-scale hydropower case studies [17] and also quantified the environmental impacts of electricity generation from three micro-hydropower case studies, using a life cycle assessment approach [18]. Su and Karney evaluated the economic feasibility of energy recovery turbines in municipal water systems, by means of a micro hydroelectric plant located in Vancouver (Canada) [19]. The feasibility of recovering waste energy from typical bio-gas upgrading facilities by means of a centrifugal pump operating in reverse flow in a specific test rig was analyzed by Bansal and Marshall in [20].

In 2018, two studies investigated the use of PATs for irrigation networks. Perez-Sanchez et al. [21] proposed a new maximization methodology for recovering energy by also considering the feasibility of the installation, while Morillo et al. [22] quantified the potential of hydropower energy recovery in a pressurized irrigation network, assessing both its technical and economic feasibility.

As highlighted by Sammartano et al. in [23] and by Carravetta et al. in [24], PATs are suitable for low and variable power, since they combine low installation costs with acceptable energy production. Indeed, pumps can be used in turbine mode by reversing the flow direction with the electric motor acting as a generator [25]. An extensive review regarding the potential benefits of PATs is documented by Nautiyal and Kumar in [26] and by Jain and Patel in [27], mainly for low capacity power generation in micro- hydropower plants, as well as in the water supply and distribution piping systems. The use of PATs may also increase the flexibility of the water distribution network, for example by changing PAT working conditions in the case of pipe failure. In fact, Venturini et al. [28] presented an energy analysis aimed at estimating the energy potential of PATs to exploit the available hydraulic energy of water distribution networks. Four pumps were tested in four water distribution networks, for which experimental data covering one year was available. By considering the actual variability of flow rate and available head over one year, four optimal combinations were identified and the consequent producible electric energy and conversion efficiency were estimated. An up-to-date, state-of-the-art review of the two most challenging PAT issues, namely PAT performance prediction and PAT flow stability aspects is presented by Binama et al. in 
[29]. A further innovative application for PATs, not reviewed in [29], is presented by Gao and Feng in [30], which investigates a circulating water system used as a cooling system in process industries. Du et al. [31] also investigated the feasibility and performance of a PAT used in a water supply system for electricity generation, by considering installation and control strategy.

The cost-effectiveness of PATs has also been recently analyzed. Novara et al. [32], for instance, compared data from 324 commercially available centrifugal pumps. The results showed that PAT unit costs ranged from 115 to $5,600 € / \mathrm{kW}$ according to their rated power. Another study by Novara et al. [33] evaluated the cost per nominal power of 280 radial end-suction PATs. Compared to conventional Francis, Pelton or Kaplan turbines, generating sets relying on PAT technology showed a cost per nominal power of up 15 times lower.

The optimal coupling between the turbomachine and available head and flow rate should also be identified by considering transient conditions, as discussed by Carravetta et al. in [24]. In particular, De Marchis et al. [34] presented the development of a mathematical model able to dynamically simulate a water distribution network including a PAT. Simani et al. and Finotti et al. studied the hydraulic system dynamic response and its optimal control in [35-38]. In this area, De Marchis et al. investigate the transient operation of PATs operating in a network characterized by intermittent distribution and by inequities among the user in terms of water supply [39].

Other issues related to the use of PATs have been investigated in the last years. Tao et al. [40] experimentally and numerically investigated the cavitation behavior in the pump mode of a pump-turbine. Abazariyan et al. [41] presented an experimental study to disclose the effects of viscosity on the performance of a PAT. Hao et al. [42] investigated transient cavitating flows in a mixed-flow PAT in pump mode, using both experimental and numerical methods. Li et al. [43] used numerical simulations to investigate the hydraulic force on the impeller of a model reversible pump turbine. With the aim of improving PAT performance, Wang et al. [44] designed a special impeller with forward-curve blades and investigated the method for determining blade inlet and outlet angles.

The main challenge related to PAT field application is that pump manufacturers do not usually provide the performance curves of pumps running in reverse mode. The designer therefore lacks data, which negatively affects the choice of the most suitable machine. Therefore, establishing a correlation that enables the transformation from pump performance curves to turbine performance curves is crucial. Many researchers have presented some theoretical and empirical relationships for predicting the Best Efficiency Point (BEP) of a PAT. Derakhshan and Nourbakhsh tested several centrifugal pumps while running as turbines and derived some relationships to predict the respective best efficiency points based on pump hydraulic characteristics. Two equations were also presented to estimate the complete characteristic curves based on their best efficiency point [45]. The same authors predicted the best efficiency point of an industrial centrifugal pump running as a turbine by using a theoretical analysis and also simulated the pump in direct and reverse modes by using a three-dimensional computational fluid dynamic model [46]. Yang et al. also coupled theoretical analysis and computational fluid dynamics to estimate the performance of a single stage centrifugal pump [47]. More recently, Derakhshan and Kasaeian have further investigated the use of computational fluid dynamic tools to optimize the geometry of the blades of an axial pump used as a propeller turbine, to achieve maximum hydraulic efficiency [48]. Fecarotta et al. investigated the use of affinity laws to predict the behavior of a machine operating at variable speeds and also proposed a new model based on relaxation of the affinity equations [49].

Huang et al. [50] presented an innovative theoretical approach to predict the flow rate and head at BEP for both pump and turbine mode, according to the principle of characteristics matching between rotor and volute. A theoretical formula regarding rotor characteristics in turbine mode was derived, based on the Euler equation of rotomachinery and velocity relations at the inlet and the outlet of the rotor. The proposed method was verified by means of the experimental results regarding three types of pumps in both pump and turbine modes.

Despite the models available in literature, a procedure to estimate their performance in turbine mode over the entire operation range has not yet been completely established in literature. In fact, experimental characterization is usually required ad hoc and case by case.

Predicting PAT curves is still an open issue due to the lack of information provided by manufacturers and the scarcity of laboratory tests that focus on this topic. Moreover, the models derived from experiments and available in literature usually refer to a specific type of pumps (e.g. semi-axial submersible single stage pumps (Fecarotta et al. in [49]) and horizontal single-stage pumps (Pugliese et al. in [51])) although different types of pumps are available on the market and are commonly used (e.g. vertical axis pumps, multi-stage pumps).

A rather new and promising field of research is the numerical study of PAT operation by means of computational fluid dynamic (CFD) simulations. In fact, a CFD model was built by Carravetta et al. in [7] based on a 3D geometrical model of the considered machine and the predicted performance curves were compared to the experimental ones. A CFD model was also developed by Buono et al. in [52] starting from the real geometry, with a commercial three-dimensional code. The aim of the paper was to investigate the possibility of using simulation methodology to obtain the inverse characteristics of a commercial centrifugal pump. The analysis presented by De Rose et al. in [53] theoretically estimates the behavior of a PAT at its BEP and extends the investigation to other operating points using both a combined theoretical approach and a CFD simulation under dynamic conditions. The effects of possible modifications to the initial design of the pump are investigated when running in turbine mode and their influence on the standard pump operation is also determined.

To the best of the authors' knowledge, in the last two years, at least six papers $[29,51,54,55,56,57]$ have specifically dealt with the prediction of PAT performance, thus representing state-of-the-art research in this scientific field.

Binama et al. [29] presented a thorough literature review of the key technical aspects related to PATs, i.e. PAT selection, performance prediction and flow stability. The main conclusions of this study are that PATs provide many advantages over conventional turbines, especially in off-grid energy systems. However, their selection for a specific site still presents difficulties as pump manufacturers do not provide reverse mode operational data for their products. Binama et al. also point out that numerous studies have been carried out on PAT performance prediction but no universal prediction method applicable to a wide range of specific speeds has yet been found.

Pugliese et al. [51] presented the laboratory results for two centrifugal pumps running in reverse mode. The uncertainty analysis highlighted that the uncertainty of flow, head, power and efficiency was equal to $\pm 0.26 \%, \pm 2.09 \%, \pm 2.93 \%$ and $\pm 3.61 \%$, respectively. Experimental data were also compared to eleven theoretical models available in literature. For the horizontal single-stage pump, results showed that the model of Derakhshan and Nourbakhsh developed in [45] is reliable even outside the investigated range (i.e. for flow rate numbers lower than 0.40 ) for head, whereas it fails to predict the generated power for greater flow rate numbers. Furthermore, the comparison between experimental data and theoretical mono-dimensional approaches highlighted how, for the considered machines, the BEP in reverse mode can be predicted with differences generally lower than $30 \%$. The results were extended to vertical two-stage centrifugal pumps, once again proving that the power curve equation provided by Derakhshan and Nourbakhsh [45] was only valid for flow rate numbers lower than 
0.40 , while both the head curve and the proposed equation for the horizontal pump underestimated experiments by up to $30 \%$.

Barbarelli et al. [54] presented a one-dimensional numerical code, with the aim of identifying the geometry and performance of a generic PAT based on passage sections and losses in each section of the machine. Starting from catalogue information and using design techniques, the one-dimensional numerical code computes a virtual geometry and then calculates fluid dynamic losses to estimate the geometrical parameters involved in the simulation. The method was validated by using laboratory test data for six PATs. By comparing the theoretical curves to some experimental measurements on PATs working at specific speeds from 9 to $65 \mathrm{rpm} \mathrm{m}^{3 / 4} \mathrm{~s}^{-1 / 2}$, the estimation error was in the range of $-5.26 \%$ to $21.36 \%$ for head at the BEP and in the range of $-21.43 \%$ to $9.30 \%$ for efficiency at the BEP.

Barbarelli et al. [56] also presented the results of an experimental and theoretical activity regarding PATs. The experimental activity dealt with twelve pumps measured both on a test rig and during normal operation. A statistical method involving polynomials was implemented, thus allowing the determination of performance curves.

Tan and Engeda [55] presented a comprehensive correlation aimed at predicting the turbine mode operation of centrifugal pumps. This work used experimental data from four pumps representing the centrifugal pump configurations in terms of specific speed and specific diameter, at the BEP of the centrifugal pump in its turbine mode operation. The estimation error at the BEP of the four considered PATs was in the range of $-7.78 \%$ to $5.19 \%$ for head, $-13.1 \%$ to $10.8 \%$ for power and $-2.86 \%$ to $4.23 \%$ for efficiency. This method was compared to nine methods found in literature. The head and flow at the BEP were precisely predicted by the method developed in [55], while all other methods had errors above $10 \%$, in some cases $20 \%$ and in others more than $80 \%$.

Rossi and Renzi [57] set up artificial neural networks to forecast both the BEP and the performance curves of PATs. Experimental data, taken from literature, were used to train the networks; their operating conditions in pump mode were the inputs, while PAT performance was the output. Artificial neural networks proved to be quite accurate. In fact, during the process, the comparison of predicted and experimental data achieved values of the coefficient of determination equal to 0.96152 at BEP and 0.98429 for BEP and performance curves.

In this framework, this paper presents the development of a physics-based simulation model, aimed at predicting the performance curves of PATs based on the performance curves of the respective pump. The simulation model implements the equations which allow the estimation of head, power and efficiency for both direct and reverse operation, in the form presented by Gulich in [58]. Moreover, the simulation model makes use of loss coefficients and specific parameters, which allow its tuning on a given machine. The simulation model is calibrated on literature data, reporting both pump and PAT performance curves over the entire range of operation. The tuning parameters are identified by means of an optimization procedure applied by using pump performance curves. The performance data, derived from literature (Derakhshan and Nourbakhsh, [45]), were taken experimentally from four different turbomachines, running in both pump and PAT mode and characterized by specific speed values in the range of 1.53-5.82. The reliability of the physics-based simulation model is tested against the respective PAT performance curves. In this paper, the predictive reliability of the simulation model is also compared to several models available in literature (not all of them physics-based) and also to that of alternative approaches in another paper by Venturini et al. [59]. Specifically, three alternative approaches are considered: two gray box models taken from literature, which integrate theory about turbomachines with specific data correlations, and an Evolutionary Polynomial Regression model, which is a black box data-driven hybrid technique, allowing the identification of an explicit mathematical relationship between PAT and pump curves.

As highlighted by the survey presented above, at present the lack of knowledge in literature is still too great to make PATs suitable for field applications, since the availability of PAT performance curves is required over the entire range of flow rate values (i.e. not only at BEP). In fact, as highlighted by Binama et al. in [29], PATs are usually applied in water and supply water distribution systems, which are typically characterized by variable discharges and head drops. To deal with these, different approaches can be used, i.e. hydraulic regulation, electric regulation or hydraulic-electric regulation [24,60]. However, regardless of the selected control strategy, PATs do not usually operate at the BEP and therefore they are usually operated in off-design conditions, as also demonstrated by Venturini et al. in [28] for PATs employed in water distribution networks. Thus, to effectively estimate the electric energy that can be produced by a PAT, it is important to accurately estimate the whole performance curve. Furthermore, in the case of electric regulation strategy, these performance curves must be known at different rotational speeds.

This paper tackles this issue, by presenting a modeling approach capable of predicting PAT performance curves over the entire range of operation (not only at the BEP), unlike most of the studies reported in literature. However, the development of a physics-based model, as for instance in the approach presented by Gulich [58] which is applied in this paper, requires detailed geometrical data that are not usually available. Therefore, this paper suggests the use of an optimization procedure to overcome this obstacle and thus identify model parameters. This avoids the use of a specific geometrical model for identifying such parameters, as proposed by Barbarelli et al. in [54].

Another novel feature of this paper is that prediction accuracy is quantitatively assessed over the entire range of operation, for all three performance curves (head, power and efficiency). This complete and quantitative analysis is rarely documented in other studies.

The reliability of model predictions is also tested in a real-world case study, by considering a water distribution network characterized by means of experimental data taken over one year, with the aim of estimating the producible electric energy.

This paper is organized as follows: the simulation model is presented in Section 2, for both pump and PAT operation; pump and PAT performance curves, derived from experimental data available in literature, are reported in Section 3; the results of the tuning and prediction capability of the simulation model are presented and discussed in Section 4 and, finally, conclusions are provided in Section 5.

\section{Simulation model}

\subsection{Pump model}

The relations implemented in the simulation model of a pump are taken from the basic theory of pumps, in the form reported by Gulich in [58]. The theoretical head of a pump can be calculated by means of the Euler's equation presented in Eq. (1):

$H_{\mathrm{th}, \mathrm{P}}=\frac{1}{g}\left(u_{2} c_{2 \mathrm{u}}-u_{1} c_{1 \mathrm{u}}\right)$

The actual head $H_{\mathrm{P}}$ can be estimated from theoretical head $H_{\text {th, }}$ by subtracting all hydraulic losses between the suction and discharge nozzles, i.e. the hydraulic losses in the inlet casing $\left(Z_{\mathrm{E}}\right)$, the impellers $\left(Z_{\mathrm{La}}\right)$, diffusers $\left(Z_{\mathrm{Le}}\right)$, volutes $\left(Z_{\mathrm{sp}}\right)$ and the outlet casing $\left(Z_{\mathrm{A}}\right)$, according to Eq. (2):

$H_{\mathrm{P}}=H_{\mathrm{th}, \mathrm{P}}-Z_{\mathrm{E}}-Z_{\mathrm{La}}-Z_{\mathrm{Le}}-Z_{\mathrm{sp}}-Z_{\mathrm{A}}$ 
Thus, the pump's hydraulic efficiency can be calculated as in Eq. (3):

$\eta_{\mathrm{h}, \mathrm{P}}=\frac{H_{\mathrm{P}}}{H_{\mathrm{th}, \mathrm{P}}}$

The useful power is defined in Eq. (4), where $Q$ is the useful flow rate:

$P_{\mathrm{u}, \mathrm{P}}=\rho g H_{\mathrm{P}} Q$

The volumetric efficiency, defined in Eq. (5), accounts for the leakage through the annular seal at the impeller inlet $Q_{\mathrm{sp}}$, the leakage $Q_{\mathrm{E}}$ through the device for axial thrust balancing and additional fluid $Q_{\mathrm{h}}$ circulated within the pump (e.g. branched off for auxiliary purposes such as feeding a hydrostatic bearing, flushing, sealing or cooling):

$\eta_{\mathrm{V}, \mathrm{P}}=\frac{Q}{Q+Q_{\mathrm{sp}}+Q_{\mathrm{E}}+Q_{\mathrm{h}}}$

Therefore, it is possible to estimate the power $P_{\mathrm{p}}$ required at the coupling by accounting for all pump losses. Power losses include mechanical power losses $P_{\mathrm{m}}$, power losses due to fluid recirculation $P_{\mathrm{Rec}}$, disk friction losses $P_{\mathrm{RR}}$, throttling losses $\mathrm{P}_{\mathrm{s} 3}$ and friction losses $P_{\mathrm{er}}$ created by the components of axial thrust balance devices. Therefore, the power $P_{\mathrm{P}}$ can be expressed as in Eq. (6):

$P_{\mathrm{P}}=\sum_{1}^{N_{\mathrm{st}}} \frac{\rho g H Q}{\eta_{\mathrm{V}} \eta_{\mathrm{h}}}+P_{\mathrm{m}}+P_{\mathrm{Rec}}+\sum_{1}^{N_{\mathrm{st}}} P_{\mathrm{RR}}+\sum_{1}^{N_{\mathrm{st}}} P_{\mathrm{s} 3}+P_{\mathrm{er}}$

Finally, the overall pump efficiency at coupling $\eta_{\mathrm{P}}$ can be estimated according to Eq. (7):

$\eta_{P}=\frac{P_{\mathrm{u}, \mathrm{P}}}{P_{\mathrm{P}}}$

$=\frac{\eta_{\mathrm{V}} \eta_{\mathrm{h}} \rho g H Q}{\rho g H Q+\eta_{\mathrm{V}} \eta_{\mathrm{h}}\left(P_{\mathrm{m}}+P_{\mathrm{Rec}}+\sum_{1}^{N_{\mathrm{st}}} P_{\mathrm{RR}}+\sum_{1}^{N_{\mathrm{st}}} P_{\mathrm{S} 3}+P_{\mathrm{er}}\right)}$

\subsection{PAT model}

This section presents the modeling approach used to simulate the behavior of a pump running in turbine mode, when the liquid transfers power to the rotor. The discharge nozzle of the pump is an inlet nozzle to the turbine, while the pump suction nozzle becomes the turbine outlet nozzle. As previously shown for pumps, the equations implemented in the simulation model of a PAT are written in the form reported by Gulich in [58].

The theoretical head of a PAT can be expressed as in Eq. (8):

$$
\begin{aligned}
H_{\mathrm{th}, \mathrm{T}} & =\frac{1}{g}\left(u_{2} c_{2 \mathrm{u}}-u_{1} c_{1 \mathrm{u}}\right) \\
& =\frac{1}{g}\left(u_{2} c_{2 \mathrm{~m}} \cot \alpha_{2}-u_{1}^{2}+u_{1} c_{1 \mathrm{~m}} \cot \beta_{1}\right)
\end{aligned}
$$

The inflow angle $\alpha_{2}$ to the runner can be determined from the guide wheel or volute geometry. The flow angle $\beta_{1}$ of the fluid exiting the runner, which can be estimated from the throat area, differs from the blade angle $\beta_{1 \mathrm{~B}}$ because a vane-congruent flow cannot be expected in turbine operation.

According to Eq. (8), the theoretical head of a PAT increases linearly with the flow rate. However, the theoretical head $H_{\text {th, }}$ transferred from the fluid to the runner is smaller than the actual head $H_{\mathrm{T}}$ between inlet and exhaust nozzles because of hydraulic losses, as shown in Eq. (9):

$H_{\mathrm{T}}=H_{\mathrm{th}, \mathrm{T}}+Z_{\mathrm{E}}+Z_{\mathrm{La}}+Z_{\mathrm{Le}}+Z_{\mathrm{sp}}+Z_{\mathrm{A}}$
The following relation applies for PAT hydraulic efficiency:

$\eta_{\mathrm{h}, \mathrm{T}}=\frac{H_{\mathrm{th}, \mathrm{T}}}{H_{\mathrm{T}}}$

As previously shown in Eq. (6), the power $P_{\mathrm{T}}$ available at the coupling of the turbine is smaller than the supplied power $\rho g H Q$, because of power losses. In fact, the power balance of a PAT can be expressed according to Eq. (11):

$$
\begin{aligned}
P_{\mathrm{T}}= & \rho g H \eta_{\mathrm{h}, \mathrm{T}}\left(Q-Q_{\mathrm{sp}}-Q_{\mathrm{E}}\right)-P_{\mathrm{m}} \\
& -\sum_{1}^{N_{\mathrm{st}}} P_{\mathrm{RR}}-\sum_{1}^{N_{\mathrm{st}}} P_{\mathrm{s} 3}-P_{\mathrm{er}}
\end{aligned}
$$

where flow rates and powers have the same meaning as in the pump model.

PAT overall efficiency $\eta_{\mathrm{T}}$ at the coupling is given by Eq. (12):

$\eta_{\mathrm{T}}=\frac{P}{\rho g H Q}$

\subsection{Model parameters}

In general, model parameters include pump geometry (e.g. impeller diameter and volute dimensions) and flow angles (e.g. $\alpha_{2}$ and $\beta_{1}$ ). Moreover, since neither the hydraulic losses nor resistance characteristics can be predicted from basic principles, the turbine characteristics are often estimated by means of statistical correlations. To this end, the performance data at maximum efficiency in turbine operation $H_{\mathrm{BEP}, \mathrm{T}}$ are related to the corresponding data at pump BEP. The ratios $H_{\mathrm{BEP}, \mathrm{T}} / H_{\mathrm{BEP}, \mathrm{P}}$ and $Q_{\mathrm{BEP}, \mathrm{T}} / Q_{\mathrm{BEP}, \mathrm{P}}$ are either related to the total efficiency or to the hydraulic efficiency, or represented as a function of the specific speed. Some examples of such functional dependencies are shown by Gulich in [58], where it is highlighted that calculating the turbine characteristics on the basis of statistical correlations is subject to considerable uncertainties (e.g. scatter values are typically $\pm 20 \%$ ), because such statistics cannot take into account the actual geometric properties of the considered machines.

An alternative approach to performance prediction from statistical data is based on loss analysis, as discussed by Gulich in [58]. This procedure consists of two steps:

- Determining the correlations for BEP flow and head from turbine test data;

- Using the correlations determined in the previous step for turbine performance prediction.

In this paper, the core methodology outlined by Gulich in [58] is implemented, with some adaptations by the authors, which mainly consist of the definition of new model parameters and improvements to the tuning procedure through an optimization process.

The parameters $x_{1}$ through $x_{24}$, which must be set in the simulation model in order to tune it on a given pump/PAT, are reported in Table 1.

Fourteen parameters are specific to the pump. In particular, the parameters $x_{1}$ through $x_{5}$ are defined as nondimensional ratios of geometric characteristics, while the parameters $x_{6}$ through $x_{11}$ identify flow and geometrical angles. Moreover, the parameters $x_{12}$ through $x_{14}$ allow the estimation of two sources of hydraulic and power losses, according to Eqs. (2) and (6). These parameters may be known from pump geometry (e.g. $x_{1}$ through $x_{11}$ ) or can be estimated through an optimization procedure as done in this paper due to a lack of detailed geometrical data.

The pump parameters $x_{1}$ through $x_{5}$ and $x_{8}$ through $x_{11}$ are also required for the setup of the PAT model. Moreover, ten additional para- 
Table 1

Simulation model parameters.

\begin{tabular}{|c|c|c|c|}
\hline & Parameter & $\begin{array}{l}\text { Meas. } \\
\text { unit }\end{array}$ & Description \\
\hline & Pump & & \\
\hline$x_{1}$ & $\mathrm{~d}_{1} / \mathrm{d}_{2}$ & - & $\begin{array}{l}\text { impeller inlet diameter/impeller outlet } \\
\text { diameter }\end{array}$ \\
\hline$x_{2}$ & $\mathrm{~b}_{2} / \mathrm{d}_{2}$ & - & $\begin{array}{l}\text { impeller outlet width/impeller outlet } \\
\text { diameter }\end{array}$ \\
\hline$x_{3}$ & $\mathrm{~d}_{3} / \mathrm{d}_{2}$ & - & volute diameter/impeller outlet diameter \\
\hline$x_{4}$ & $\mathrm{~b}_{3} / \mathrm{b}_{2}$ & - & volute width/impeller outlet width \\
\hline$x_{5}$ & $\mathrm{~s}_{\mathrm{ax}} / \mathrm{d}_{2}$ & - & $\begin{array}{l}\text { axial casing clearance/impeller outlet } \\
\text { diameter }\end{array}$ \\
\hline$x_{6}$ & $\varepsilon_{\mathrm{sp}}$ & degree & wrap angle of the inner volute \\
\hline$x_{7}$ & $\alpha_{1}$ & degree & flow angle at impeller inlet \\
\hline$x_{8}$ & $\beta_{2 \mathrm{~B}}$ & degree & blade angle at impeller outlet \\
\hline$x_{9}$ & $\beta_{1 \mathrm{~B}}$ & degree & blade angle at impeller inlet \\
\hline$x_{10}$ & $\alpha_{3 \mathrm{~B}}$ & degree & volute cutwater camber angle \\
\hline$x_{11}$ & $\lambda$ & degree & angle between vanes and side disks \\
\hline$x_{12}$ & $\varsigma_{\mathrm{E} 1}$ & - & inlet casing loss coefficient \#1 \\
\hline$x_{13}$ & $\varsigma_{\mathrm{E} 2}$ & - & inlet casing loss coefficient \#2 \\
\hline \multirow[t]{2}{*}{$x_{14}$} & $\mathrm{y}_{\mathrm{er}, \mathrm{P}}$ & - & parameter for estimating $P_{\mathrm{er}}$ in a pump \\
\hline & PAT & & \\
\hline$x_{15}$ & $\phi_{\mathrm{BEP}, \mathrm{T}} / \phi_{\mathrm{BEP}, \mathrm{P}}$ & - & Ratio of PAT/pump flow rate at the BEP \\
\hline$x_{16}$ & $\psi_{\mathrm{BEP}, \mathrm{T}} / \psi_{\mathrm{BEP}, \mathrm{P}}$ & - & Ratio of PAT/pump head at the BEP \\
\hline$x_{17}$ & SLa,BEP & - & PAT losses at the BEP \\
\hline$x_{18}$ & $S_{\text {Le,BEP }}$ & - & PAT diffuser or volute losses at the BEP \\
\hline$x_{19}$ & $\mathrm{y}_{\mathrm{z}}$ & - & parameter for estimating hydraulic losses \\
\hline$x_{20}$ & $\mathrm{y}_{\mathrm{S} 3,1}$ & - & $\begin{array}{l}\text { parameter \#1 for estimating throttling losses } \\
\mathrm{P}_{\mathrm{s} 3}\end{array}$ \\
\hline$x_{21}$ & $\mathrm{y}_{\mathrm{S} 3,2}$ & - & $\begin{array}{l}\text { parameter \#2 for estimating throttling losses } \\
\mathrm{P}_{\mathrm{s} 3}\end{array}$ \\
\hline$x_{22}$ & $\mathrm{y}_{\mathrm{er}, \mathrm{T}}$ & - & parameter for estimating $P_{\mathrm{er}}$ \\
\hline$x_{23}$ & $\mathrm{y}_{\mathrm{u}, 1}$ & - & $\begin{array}{l}\text { parameter \#1 for estimating the useful } \\
\text { power P }\end{array}$ \\
\hline$x_{24}$ & $\mathrm{y}_{\mathrm{u}, 2}$ & - & $\begin{array}{l}\text { parameter \#2 for estimating the useful } \\
\text { power } \mathrm{P}\end{array}$ \\
\hline
\end{tabular}

meters are required for tuning the simulation model on the corresponding PAT. Parameters $x_{15}$ and $x_{16}$ allow the identification of the BEP of the PAT, in terms of flow rate and head with respect to the corresponding values for the pump (which are known from pump performance curves). Parameters $x_{17}$ through $x_{19}$ refer to hydraulic losses, while parameters $x_{20}$ through $x_{24}$ are used to tune power losses (see Eq. (11)) and calculate the value of the useful power accordingly.

\subsection{Tuning procedure}

To identify the optimal value of the parameters listed in Table 1, which allow the tuning of the simulation model on a given pump and its corresponding PAT, an optimization procedure was adopted, making use of the tool Optimtool [61] and a solver called fmincon, both available in Matlab®. This solver can search the minimum value of a scalar Objective Function (OF).

To this end, the Root Mean Square Relative Error RMSE $\mathrm{Yk}_{\mathrm{Yk}}$ can be adopted according to Eq. (13):

$$
\begin{aligned}
\operatorname{RMSE}_{\mathrm{Yk}} & =\sqrt{\frac{1}{\mathrm{~N}_{\mathrm{e}}} \sum_{\mathrm{i}=1}^{\mathrm{N}_{\mathrm{e}}}\left(\frac{\left(\mathrm{Y}_{\mathrm{ki}}\right)_{\mathrm{e}}-\left(\mathrm{Y}_{\mathrm{ki}}\right)_{\mathrm{s}}}{\left(\mathrm{Y}_{\mathrm{ki}}\right)_{\mathrm{e}}}\right)^{2}} Y \\
& =\psi, \pi, \eta ; k \\
& =1, \ldots, N_{\mathrm{P}, \mathrm{T}}
\end{aligned}
$$

The RMSE $E_{\mathrm{Yk}}$ compares the experimental values of the performance parameter $\left(Y_{\mathrm{ki}}\right)_{\mathrm{e}}$ for the $k$-th pump/PAT (reported by Derakhshan and Nourbakhsh in [45]) to the corresponding simulated value $\left(Y_{\mathrm{ki}}\right)_{s}$, predicted by means of the physics-based model developed in this paper for all the available $N_{\mathrm{P}, \mathrm{T}}$ pumps. This index will be used for assessing the reliability of the physics-based model.
For the purpose of model tuning, RMSE $\mathrm{Ykint}_{\text {is }}$ also defined according to Eq. (14):

$$
\begin{aligned}
\operatorname{RMSE}_{\mathrm{Ykint}} & =\sqrt{\frac{1}{\mathrm{~N}_{\mathrm{int}}} \sum_{\mathrm{i}=1}^{\mathrm{N}_{\mathrm{int}}}\left(\frac{\left(\mathrm{Y}_{\mathrm{ki}}\right)_{\mathrm{int}}-\left(\mathrm{Y}_{\mathrm{ki}}\right)_{\mathrm{s}}}{\left(\mathrm{Y}_{\mathrm{ki}}\right)_{\mathrm{int}}}\right)^{2}} Y \\
& =\psi, \pi, \eta ; k \\
& =1, \ldots, N_{\mathrm{P}, \mathrm{T}}
\end{aligned}
$$

Unlike RMSE $E_{Y k}, R_{M S E} E_{\text {Ykint }}$ compares the values on the interpolation curves derived by Venturini et al. in [28] (Eqs. (16) and (17)), sampled on $N_{\text {int }}$ operating points of the performance parameter $\left(Y_{\mathrm{ki}}\right)_{\text {int }}$ for the $k$-th pump/PAT to the corresponding simulated value $\left(Y_{\mathrm{ki}}\right)_{s}$, predicted by the physics-based model. In fact, a greater number of available operating points (i.e. $N_{\text {int }}$ instead of $N_{\mathrm{e}}$, which in [45] is lower than 20 for a given performance curve at a given specific speed) allows a more robust convergence of the OF defined in Eq. (15):

$$
\begin{aligned}
\mathrm{OF} & =R M S E_{\psi \mathrm{kint}}+R M S E_{\pi \mathrm{kint}}+R M S E_{\eta \mathrm{kint}} \quad k \\
& =1, \ldots, N_{\mathrm{P}, \mathrm{T}}
\end{aligned}
$$

The value of the OF depends on the values of model parameters listed in Table 1 and, obviously, the lower the value, the better.

The tuning procedure, outlined in Fig. 1, consists of two steps. First, the simulation model is tuned in order to simulate pump behavior. The required inputs are (i) pump geometrical and operational information (e.g. impeller outlet diameter $\mathrm{d}_{2}$ and pump volumetric efficiency $\eta_{\mathrm{V}, \mathrm{P}}$ ), (ii) pump operating point (rotational speed and flow rate and head at pump best efficiency point) and (iii) the complete pump performance curves which have to be reproduced by the simulation model. In this manner, model parameters $x_{1}$ through $x_{14}$ are identified by means of the optimization algorithm. Then, by using pump parameters $x_{1}$ through $x_{5}$ and $x_{8}$ through $x_{11}$, which make PAT geometry consistent with pump geometry and by providing PAT rotational speed and PAT complete performance curves, the same optimization algorithm is also used to identify the optimal values of the remaining ten parameters for the PAT ( $x_{16}$ through $x_{24}$ ).

\section{Pump and PAT data}

\subsection{Field data available in literature}

The field data considered in this paper for deriving pump and PAT performance curves over the entire range of operation are derived from the paper [45], authored by Derakhshan and Nourbakhsh and also used for the analyses carried out by Venturini et al. in [28]. Derakhshan and Nourbakhsh [45] reported the performance data acquired experimentally from four different centrifugal pumps, running in both pump and PAT mode. The four pumps are characterized by $\Omega$ values in the range of 1.53-5.82.

The pump characteristic values at the respective BEP are reported in Table 2. As done by Venturini et al. in [28], the experimental nondimensional data reported by Derakhshan and Nourbakhsh in [45] were rendered dimensional by considering $n$ equal to $25 \mathrm{rps}$ (i.e. $1500 \mathrm{rpm}$ ) and pump nominal diameter $\mathrm{D}$ equal to $0.25 \mathrm{~m}$. Therefore, the values in Table 2 represent the experimental BEP, documented by the measured values reported by Derakhshan and Nourbakhsh in [45].

It can be seen that the four pumps are characterized by considerably different power values, from about $3 \mathrm{~kW}$ to about $22 \mathrm{~kW}$. Accordingly, the volume flow rate at the BEP passes from 8.01/s to $107.71 / \mathrm{s}$ and the maximum efficiency increases from $64.5 \%$ to $86.8 \%$. The head at the BEP decreases from $24.9 \mathrm{~m}$ to $18.3 \mathrm{~m}$, passing from pump \#1 to pump \#4. 


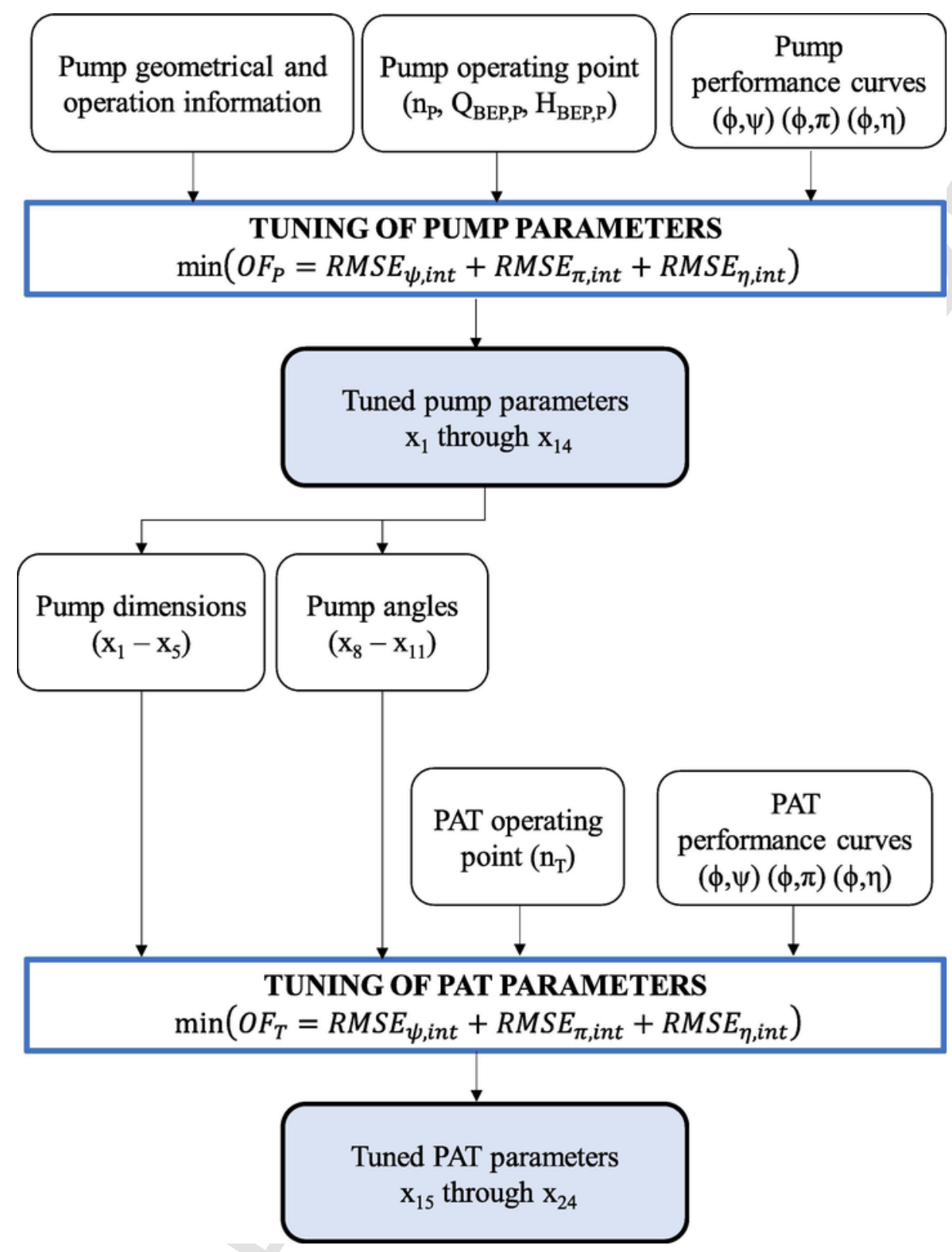

Fig. 1. Tuning procedure of the simulation model.

Table 2

Pump characteristics at the BEP [28].

\begin{tabular}{llllll}
\hline Pump & $\Omega,-$ & $Q, 10^{-3} \mathrm{~m}^{3} / \mathrm{s}$ & $\eta, \%$ & $H, \mathrm{~m}$ & $P, \mathrm{~kW}$ \\
\hline$\# 1$ & 1.53 & 8.0 & 64.5 & 24.9 & 3.044 \\
$\# 2$ & 2.41 & 24.8 & 75.7 & 22.1 & 7.114 \\
$\# 3$ & 3.94 & 62.2 & 86.3 & 21.1 & 14.926 \\
$\# 4$ & 5.82 & 107.7 & 86.8 & 18.3 & 22.288 \\
\hline
\end{tabular}

Table 3 reports the operating ranges of the four pumps/PATs. The four pumps can swallow up to 1481 s (pump \#4), with a maximum head of $29 \mathrm{~m}$ (pump \#1).

The maximum power consumption is about $25 \mathrm{~kW}$ and maximum efficiency is about $87 \%$, with both values referring to pump \#4.

Instead, the maximum volume flow rate allowed for PAT operation is slightly lower than that of the pump (i.e. up to 1291/s for PAT \#4). The required head is rather different for the four PATs, e.g. it is in the
Table 3

Pump and PAT operating range reported in [28].

\begin{tabular}{lllll}
\hline & $Q, 10^{-3} \mathrm{~m}^{3} / \mathrm{s}$ & $\eta, \%$ & $H, \mathrm{~m}$ & $P, \mathrm{~kW}$ \\
\hline Pump & & & & \\
$\# 1$ & $0.0-12.7$ & $40.1-64.5$ & $15.3-29.2$ & $1.559-2.525$ \\
$\# 2$ & $0.0-43.9$ & $30.0-75.7$ & $10.9-24.7$ & $4.084-9.504$ \\
$\# 3$ & $0.0-94.4$ & $30.3-86.3$ & $12.5-25.5$ & $8.465-18.860$ \\
$\# 4$ & $0.0-148.3$ & $30.0-86.8$ & $12.4-23.1$ & $14.405-24.800$ \\
PAT & & & & \\
$\# 1$ & $10.4-18.3$ & $24.8-63.1$ & $27.0-67.7$ & $668-4.752$ \\
$\# 2$ & $19.5-43.5$ & $25.1-71.6$ & $19.8-50.8$ & $817-15.296$ \\
$\# 3$ & $31.5-95.9$ & $0.0-74.7$ & $15.4-38.7$ & $0-26.582$ \\
$\# 4$ & $55.2-129.3$ & $0.0-78.3$ & $12.1-27.1$ & $0-25.468$ \\
\hline
\end{tabular}

range of $27-68 \mathrm{~m}$ for PAT \#1 and $12-27 \mathrm{~m}$ for PAT \#4. The maximum producible electric power passes from less than $5 \mathrm{~kW}$ (PAT \#1) to more than $25 \mathrm{~kW}$ (PAT \#4). Moreover, as expected, the maximum value of efficiency in PAT mode is lower than the maximum efficiency in pump 
mode. Such a difference ranges from $1.4 \%$ to $11.6 \%$, depending on the considered turbomachine.

\subsection{Pump and PAT performance curves}

In this paper, according to the procedure adopted by Venturini et al. in [28], pump and PAT experimental curves are modelled independently, by interpolating the available experimental data reported by Derakhshan and Nourbakhsh in [45]. Venturini et al. [28] proved that second-order polynomial functions provide the best fit for all the nondimensional parameters $Y$, for both the pump and the PAT, according to Eqs. (16) and (17), respectively. This representation also leads to physically consistent modeling over the entire range of operation.

$Y_{p}(\Omega, \phi)=a_{2 P}(\Omega) \cdot \phi^{2}+a_{1 P}(\Omega) \cdot \phi+a_{0 P}(\Omega)$

$Y_{P A T}(\Omega, \phi)=a_{2 T}(\Omega) \cdot \phi^{2}+a_{1 T}(\Omega) \cdot \phi+a_{0 T}(\Omega)$

The interpolation curves expressed in Eqs. (16) and (17) are shown in Fig. 2, taken from [28]. In these figures, the nondimensional flow rate is assumed positive for both pump and PAT. As discussed by Venturini et al. in [28], the agreement between the experimental data and interpolation curves of head is very good in both pump and PAT mode (in the range of $0.5 \%-2.6 \%$ ), while the deviation is slightly higher for the power curves of PATs (values in the range of 1.9-7.5\%). Therefore, this also reflects on efficiency curves. However, it should be noted that, according to the analyses carried out by Derakhshan and Nourbakhsh in [45], the uncertainties of the experimental data of flow rate, head, power and efficiency were $\pm 3.4 \%, \pm 5.5 \%, \pm 5.1 \%$ and $\pm 5.5 \%$, respectively. Therefore, the selected interpolating functions expressed in Eqs. (16) and (17) can be considered satisfactory, since they provide a physics-responding approach for reproducing the behavior of pumps and PATs over the entire range of operation.

\section{Results}

\subsection{Tuning of the simulation model}

The values of model parameters derived by means of the optimization procedure outlined in Fig. 1 are listed in Table 4 for pump/PAT \#1 through \#4, together with the respective optimization spaces. As can be seen, some of the optimized values reported in Table 4 are equal to one of the two extremes of the optimization space. Such parameters refer to pump geometry (e.g. $x_{1}$ and $x_{3}$ ) or to PAT losses (e.g. $x_{17}, x_{18}, x_{20}$ and $x_{21}$ ), which are physically constrained. In any case, it should be noted that the optimization space was defined according to (i) physical interpretation of each parameter and (ii) statistical correlations and rules of thumb reported by Gulich in [58].

Moreover, it is worth noting that, in practice, the optimal values reported in Table 4 are independent of the initial guess value, which was selected as the average value of the two bounds. Moreover, the influence of the number $N_{\text {int }}$ of operating points selected on the interpolation curves was analyzed. The choice of $N_{\text {int }}$ equal to 1000 proved a good compromise between optimization convergence and computational time.

Since the four pumps are characterized by specific speed values in the range of 1.53-5.82, the model parameters reported in Table 4 may represent a guideline for setting up the simulation model in order to predict PAT curves in cases where only pump performance curves are available.
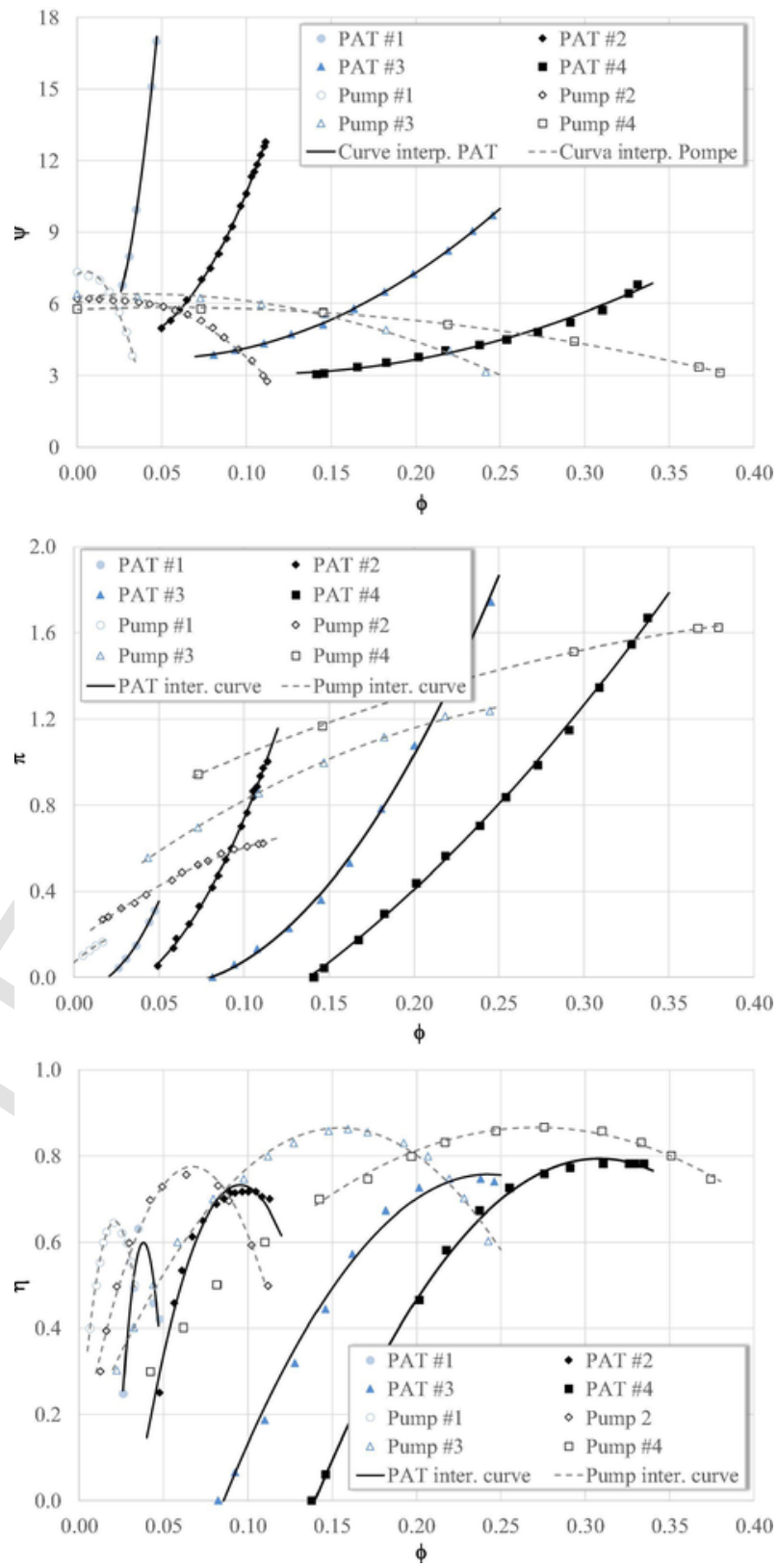

Fig. 2. Nondimensional head $\psi$ (top), nondimensional power $\pi$ (center) and efficiency $\eta$ (bottom) vs. nondimensional volume flow rate $\phi$ (symbols: experimental data reported in [45]; lines: interpolation curves derived in [28]).

\subsection{Performance curves predicted by the simulation model}

The performance curves predicted by the simulation model for both pump and PAT are reported in Figs. 3, 4 and 5 for nondimensional head, nondimensional power and efficiency, respectively. The same figures also report the original field data documented by Derakhshan and Nourbakhsh in [45]. It can be observed that:

- all the performance curves are physically sound over the entire range of operation;

- the head of pumps is usually reproduced by the simulation model by means of an almost linear curve, while the head of PAT has an increasing slope as the flow rate increases, as expected. From a qualitative point of view, both pump and PAT trends are well reproduced, but PAT behavior seems to be approximated with higher accuracy. In 
Table 4

Simulation model tuning.

\begin{tabular}{|c|c|c|c|c|c|c|c|}
\hline & Parameter & Meas. unit & Range & \multicolumn{3}{|c|}{ Optimal value } & \\
\hline & \multicolumn{3}{|l|}{ Pump } & $\# 1$ & $\# 2$ & $\# 3$ & $\# 4$ \\
\hline$x_{1}$ & $\mathrm{~d}_{1} / \mathrm{d}_{2}$ & - & {$[0.1 ; 1.0]$} & 0.100 & 0.100 & 0.100 & 0.168 \\
\hline$x_{2}$ & $\mathrm{~b}_{2} / \mathrm{d}_{2}$ & - & {$[0.1 ; 1.0]$} & 0.100 & 0.101 & 0.185 & 0.260 \\
\hline$x_{3}$ & $\mathrm{~d}_{3} / \mathrm{d}_{2}$ & - & {$[1.04 ; 2.00]$} & 1.665 & 1.040 & 1.040 & 1.040 \\
\hline$x_{4}$ & $\mathrm{~b}_{3} / \mathrm{b}_{2}$ & - & {$[1.0 ; 2.0]$} & 1.665 & 1.000 & 1.000 & 1.000 \\
\hline$x_{5}$ & $\mathrm{~s}_{\mathrm{ax}} / \mathrm{d}_{2}$ & - & {$[0.0 ; 0.5]$} & 0.022 & 0.026 & 0.343 & 0.029 \\
\hline$x_{6}$ & $\varepsilon_{\mathrm{sp}}$ & degree & {$[90 ; 180]$} & 135.382 & 138.474 & 135.033 & 134.994 \\
\hline$x_{7}$ & $\alpha_{1}$ & degree & {$[0 ; 90]$} & 14.131 & 44.324 & 44.526 & 44.68 \\
\hline$x_{8}$ & $\beta_{2 \mathrm{~B}}$ & degree & {$[0 ; 90]$} & 35.401 & 39.267 & 37.622 & 42.576 \\
\hline$x_{9}$ & $\beta_{1 \mathrm{~B}}$ & degree & {$[0 ; 90]$} & 43.699 & 44.654 & 43.632 & 42.833 \\
\hline$x_{10}$ & $\alpha_{3 \mathrm{~B}}$ & degree & {$[0 ; 90]$} & 45.004 & 44.441 & 44.757 & 44.24 \\
\hline$x_{11}$ & $\lambda$ & degree & {$[0 ; 90]$} & 44.055 & 45.055 & 45.084 & 44.899 \\
\hline$x_{12}$ & $\varsigma_{\mathrm{E} 1}$ & - & {$[0.0 ; 1.0]$} & 0.001 & 0.129 & 0.018 & 0.001 \\
\hline$x_{13}$ & $\varsigma_{\mathrm{E} 2}$ & - & {$[0.6 ; 0.7]$} & 0.600 & 0.700 & 0.700 & 0.700 \\
\hline \multirow[t]{2}{*}{$x_{14}$} & $\mathrm{y}_{\mathrm{er}, \mathrm{P}}$ & - & {$[1.0 ; 5.0]$} & 1.811 & 2.000 & 2.016 & 2.111 \\
\hline & \multicolumn{3}{|l|}{ PAT } & $\# 1$ & $\# 2$ & $\# 3$ & $\# 4$ \\
\hline$x_{15}$ & $\phi_{\mathrm{BEP}, \mathrm{T}} / \phi_{\mathrm{BEP}, \mathrm{P}}$ & - & {$[1.15 ; 2.00]$} & 1.900 & 1.478 & 1.712 & 1.150 \\
\hline$x_{16}$ & $\psi_{\mathrm{BEP}, \mathrm{T}} / \psi_{\mathrm{BEP}, \mathrm{P}}$ & - & {$[1.2 ; 2.0]$} & 1.412 & 2.000 & 2.000 & 1.883 \\
\hline$x_{17}$ & $\varsigma_{\mathrm{LL}, \mathrm{BEP}}$ & - & {$[0 ; 0.08]$} & 0.080 & 0.080 & 0.080 & 0.080 \\
\hline$x_{18}$ & SLe,BEP & - & {$[0 ; 0.2]$} & 0.200 & 0.200 & 0.200 & 0.055 \\
\hline$x_{19}$ & $\mathrm{y}_{\mathrm{z}}$ & - & {$[0.5 ; 6.0]$} & 5.423 & 3.992 & 3.480 & 1.961 \\
\hline$x_{20}$ & $\mathrm{y}_{\mathrm{S} 3,1}$ & - & {$[1.1 ; 1.5]$} & 1.100 & 1.100 & 1.100 & 1.100 \\
\hline$x_{21}$ & $\mathrm{y}_{\mathrm{S} 3,2}$ & - & {$[0.8 ; 1.2]$} & 0.800 & 0.800 & 0.800 & 0.836 \\
\hline$x_{22}$ & $\mathrm{y}_{\mathrm{er}, \mathrm{T}}$ & - & {$[0 ; 5.0]$} & 0.313 & 0.011 & 0.011 & 0.222 \\
\hline$x_{23}$ & $\mathrm{y}_{\mathrm{u}, 1}$ & - & {$[0 ; 6.0]$} & 1.554 & 2.827 & 4.415 & 5.245 \\
\hline$x_{24}$ & $\mathrm{y}_{\mathrm{u}, 2}$ & - & {$[0.5 ; 10.0]$} & 9.193 & 3.099 & 1.957 & 1.521 \\
\hline
\end{tabular}
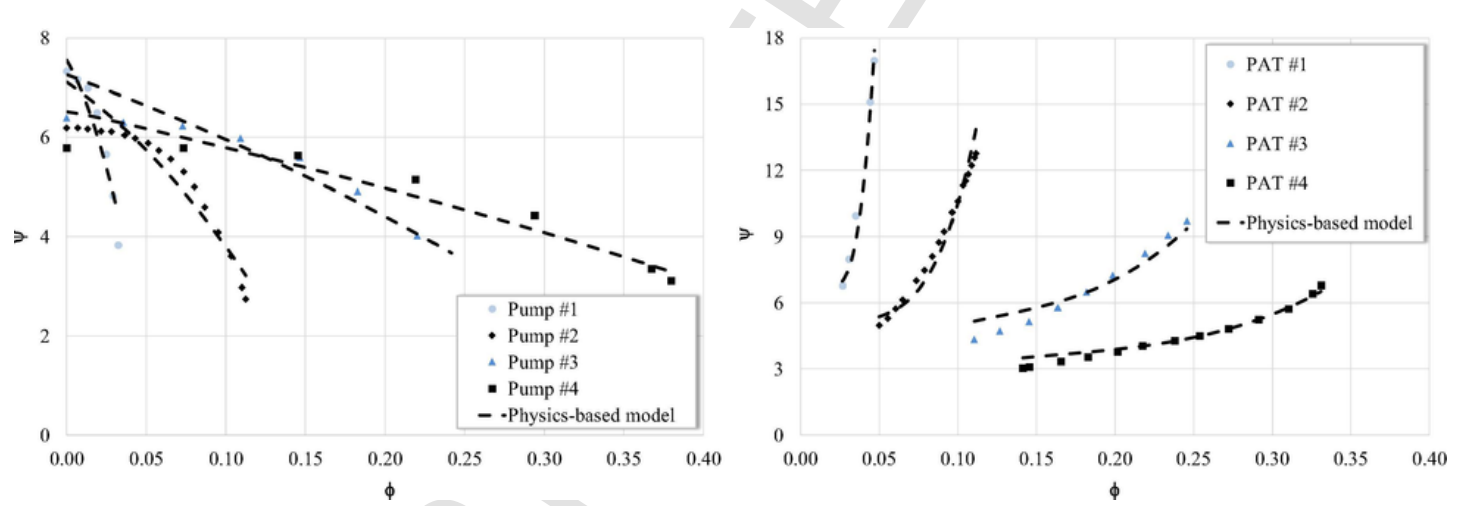

Fig. 3. Nondimensional head $\psi$ vs. nondimensional volume flow rate $\phi$ for pump (left) and PAT (right) (symbols: experimental data reported in [45]; dashed lines: simulation model).
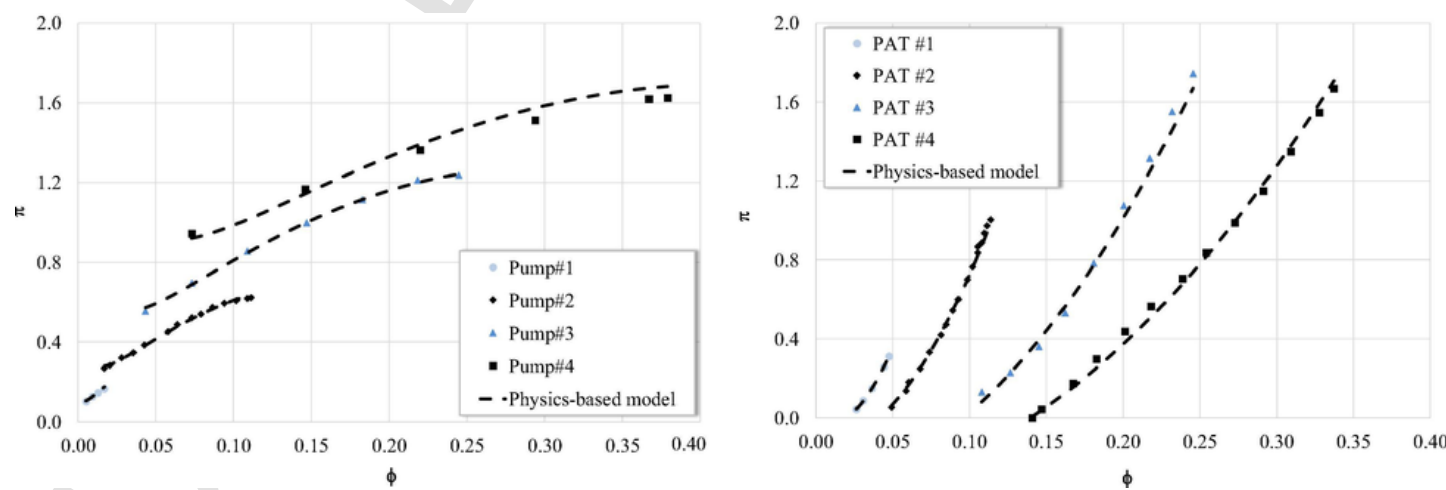

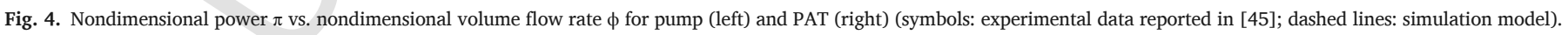
particular, the predicted curve of PAT \#1 almost overlaps the experimental data. The quantitative assessment of model prediction reliability is reported in Section 4.3;

- power is also qualitatively reproduced well in all cases, even though the trend of both pump and PAT differs slightly from the experimental data by increasing the specific speed (i.e. by passing from pump/PAT \#1 to pump/PAT \#4);

- as a consequence of the comments above regarding the head and power trends, the shape of the efficiency curves is also physically sound for all pumps/PATs. However, the efficiency curves of pumps \#3 and \#4 may only be acceptable at low flow rates, while they deviate considerably from experimental data at high flow rates and also at the respective $\mathrm{BEP}$. 

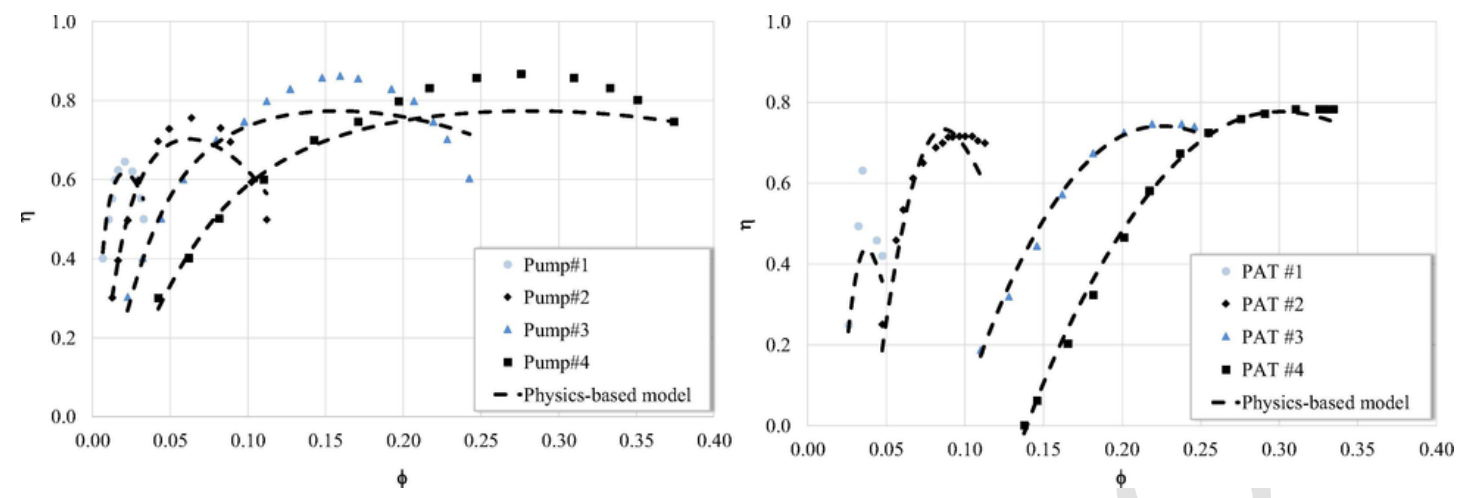

Fig. 5. Efficiency $\eta$ vs. nondimensional volume flow rate $\phi$ for pump (left) and PAT (right) (symbols: experimental data reported in [45]; dashed lines: simulation model).

\subsection{Accuracy of the simulation model}

The accuracy of pump and PAT curve prediction is assessed with respect to the original field data reported by Derakhshan and Nourbakhsh in [45]. However, it is worth noting that the prediction errors over the entire range of operation were not reported by Derakhshan and Nourbakhsh in [45]. The values of RMSE ${ }_{\mathrm{Yk}}$ are summarized in Figs. 6 and 7 for pumps and PATs, respectively. It should be noted that for PAT \#3 and \#4, the respective operating points at the lowest volume flow rate were not considered for calculating RMSE, since both power and efficiency were equal to zero.

In agreement with the comments concerning the performance curve trends made in Section 4.2, it can be highlighted that:

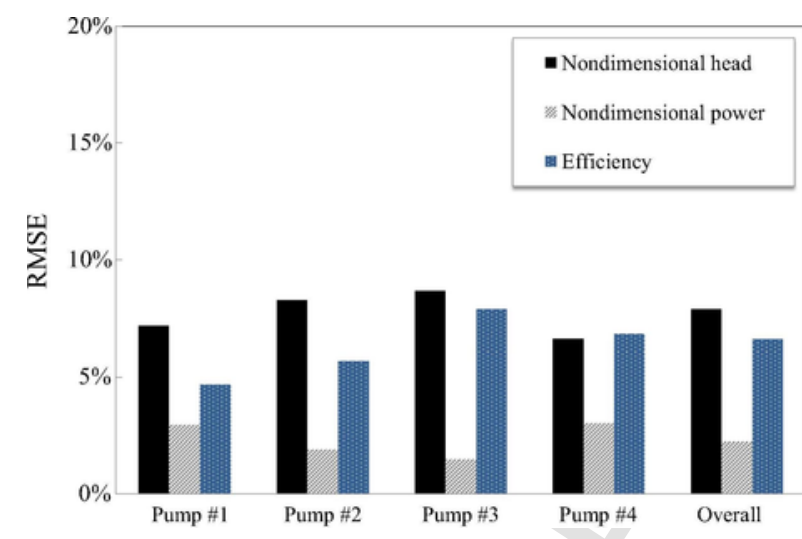

Fig. 6. Root Mean Square Relative Error (RMSE) for pumps.

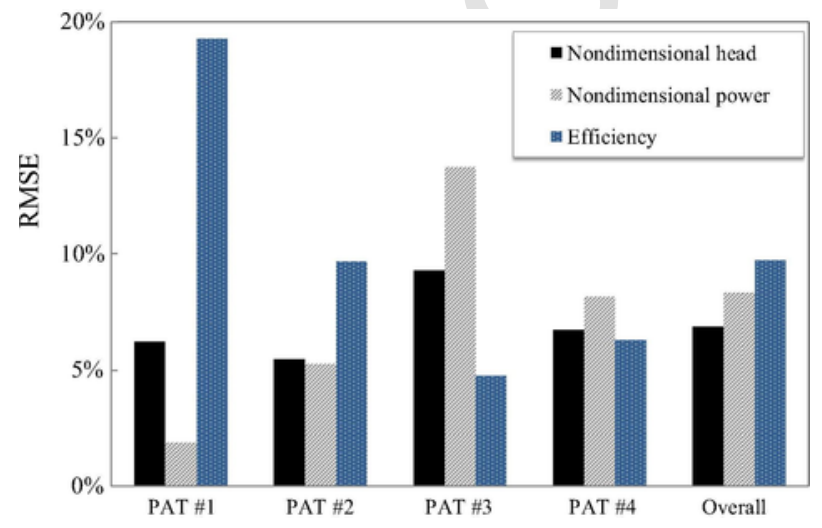

Fig. 7. Root Mean Square Relative Error (RMSE) for PATs.
- the RMSE values are almost independent of the considered pump (see Fig. 6). Moreover, the RMSE on power is considerably lower than the RMSE on head and efficiency. In fact, the overall RMSE is equal to $7.9 \%, 2.2 \%$ and $6.6 \%$ for head, power and efficiency, respectively;

- the RMSE on the head of PATs is quite acceptable (in fact, it ranges from 5.5\% to 9.3\%, according to Fig. 7), although it is more sensitive to the considered PAT.

- the RMSE on the power of PATs is more case-sensitive, passing from 1.9\% for PAT \# 1 to $13.8 \%$ for PAT \#3;

- as a consequence of the combined effect of RMSE values on head and power, the RMSE on the efficiency of PATs is usually higher than the efficiency of the respective pump for all PATs and decreases from 19.3\% (PAT \#1) to 6.3\% (PAT \#4) by increasing the specific speed.

The accuracy of model prediction has also been assessed in correspondence of the BEP. To this end, Table 5 reports the relative deviation of model prediction with respect to field data at the BEP. Such a deviation from field data at the BEP is in the following ranges:

$4.4 \%$ to $6.4 \%$ for pump head, $-3.8 \%$ to $0.9 \%$ for pump power and $4.9 \%$ to $10.8 \%$ for pump efficiency;

- $2.6 \%$ to $10.8 \%$ for PAT head, $-1.7 \%$ to $4.8 \%$ for PAT power and $1.6 \%$ to $27.1 \%$ for PAT efficiency.

Therefore, the relative deviation of head and power at the BEP always seems acceptable, since the uncertainty of the experimental data of head and power was $\pm 5.5 \%$ and $\pm 5.1 \%$, respectively [45]. Moreover, in the same paper by Derakhshan and Nourbakhsh [45], the relative deviation of the ratio of PAT head at the BEP to pump head at the $\mathrm{BEP}\left(\psi_{\mathrm{BEP}, \mathrm{T}} / \psi_{\mathrm{BEP}, \mathrm{P}}\right)$ was in the range of $-4.4 \%$ to $4.6 \%$. The relative deviation at the BEP of the other methods reviewed in [45] varied in the range of $-7.30 \%$ to $28.3 \%$.

Instead, for PATs \#2 through \#4, the relative deviation of model prediction of efficiency at the BEP is considerably lower (in the range

Table 5

Model relative deviation at the BEP.

\begin{tabular}{llll}
\hline & $\psi$ & $\pi$ & $\eta$ \\
\hline Pump & & & \\
$\# 1$ & $5.7 \%$ & $-1.0 \%$ & $4.9 \%$ \\
$\# 2$ & $5.3 \%$ & $0.9 \%$ & $9.5 \%$ \\
$\# 3$ & $4.4 \%$ & $0.0 \%$ & $10.6 \%$ \\
$\# 4$ & $6.4 \%$ & $-3.8 \%$ & $10.8 \%$ \\
PAT & & & \\
$\# 1$ & $10.8 \%$ & $0.0 \%$ & $27.1 \%$ \\
$\# 2$ & $4.0 \%$ & $1.0 \%$ & $2.5 \%$ \\
$\# 3$ & $4.8 \%$ & $4.8 \%$ & $1.6 \%$ \\
$\# 4$ & $2.6 \%$ & $-1.7 \%$ & $2.2 \%$ \\
\hline
\end{tabular}


of 1.6-2.5\%) than RMSE values over the entire operating range, thanks to the optimal tuning of model parameters $x_{15}$ and $x_{16}$, which constrains the simulation model to reproduce PAT behavior at the BEP. The contrary occurs for PAT \#1, characterized by a relative deviation of efficiency at the BEP equal to 27.1\%, as clearly highlighted in Fig. 5.

As a basis for comparison, it should be observed that the estimation errors at the BEP reported by Barbarelli et al. in [54] were also considerable, i.e. in the range of $-5.26 \%$ to $21.36 \%$ for head and $-21.43 \%$ to $9.30 \%$ for efficiency. Moreover, the estimation error at the BEP of the four PATs considered by Tan and Engeda in [55] was in the range of $-7.78 \%$ to $5.19 \%$ for head, $-13.1 \%$ to $10.8 \%$ for power and $-2.86 \%$ to $4.23 \%$ for efficiency. All the other methods analyzed by Tan and Engeda in [55] had errors for head and flow at the BEP which were above $10 \%$, in some cases $20 \%$ and in a few cases more than $80 \%$ error. Therefore, the simulation model developed in this paper proves to be in line with (and in some cases even more accurate than) other methods documented by Binama et al. in [29]. However, it should be considered that the simulation model developed in this paper allows the estimation of the complete performance curve of a pump running in turbine mode.

As a case study aimed at evaluating the capability of the physics-based model for real-world applications, the producible electric energy estimated by Venturini et al. in [28] by considering the experimental performance curves of PAT \#2 (derived by Derakhshan and Nourbakhsh in [45]), is compared to the estimate which can be obtained by using the performance curves of the same PAT \#2 predicted by the physics-based model developed in this paper and reported in Figs. 3 through 5 .

The selected case study is a water distribution network characterized by means of experimental data taken over one year. The available measured data of available head drop vs. volume flow rate are shown in Fig. 8. As can be seen, the flow rate of the majority of data is included in the range of 10-501/s, which represents a good fit for the PAT \#2 operating range reported in Table 3.

The producible electric energy, estimated by Venturini et al. in [28] by considering experimental performance curves, was equal to $40,036 \mathrm{kWh}$. Instead, the producible electric energy obtained by using the performance curves of the same PAT predicted by the physics-based model is $40,428 \mathrm{kWh}$. Therefore, the relative deviation is just $1.0 \%$. This makes the predictions of the physics-based model extremely accurate, e.g. to evaluate the energy and economic feasibility of PATs.

\section{Conclusions}

This paper presents the development of a physics-based simulation model, aimed at predicting the performance curves of PATs on the basis of the performance curves of the respective pump. The simulation model was calibrated on experimental data taken from literature, re-

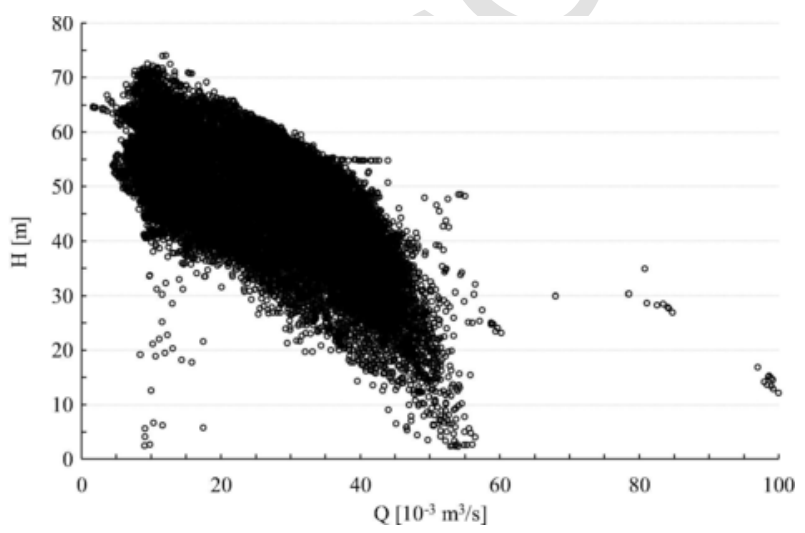

Fig. 8. Available head drop vs. volume flow rate in a water distribution network [28]. porting both pump and PAT performance curves for head, power and efficiency over the entire range of operation on four different turbomachines characterized by specific speed values in the range of 1.53-5.82.

The main achievements of this paper can be summarized as follows:

all the performance curves predicted by the simulation model are physically consistent over the entire range of operation;

the relative deviation of model prediction with respect to the field data of head and power at the BEP always seems acceptable compared to the typical deviations of other methods available in literature;

the physics-based approach adopted in this paper may also be used for the prediction of PAT curves different from the ones considered in this study for tuning the simulation model;

the simulation model represents a practical tool for identifying the most suitable pump/PAT to be installed and for the preliminary estimation of the producible electric energy in a given installation site, characterized through variable-over-time flow rate and head drop.

In conclusion, the physics-based simulation model developed in this paper represents a powerful and reliable tool for estimating PAT performance curves over the entire range of operation based on pump characteristics. In fact, the results can be considered promising, since (i) published data regarding the performance curves of pumps running in reverse mode are limited, (ii) no single modeling approach available in literature reproduces the experimental behavior of PATs over the whole range of operation, and (iii) the magnitude of prediction errors of other simulation models documented in literature is often higher. Moreover, model parameters, estimated through an optimization procedure, may provide a further insight into pump/PAT operation and, in addition to performance prediction, may also be used at a design stage.

\section{References}

[1] K.S. Balkhair, K. Ur Rahman, Sustainable and economical small-scale and low-head hydropower generation: A promising alternative potential solution for energy generation at local and regional scale, Appl Energy 188 (2017) 378-391.

[2] B.C. Kurse, D.C. Baruah, P.K. Bordoloi, S.C. Patra, Assessment of hydropower potential using GIS and hydrological modeling technique in Kopili River basin in Assam (India), Appl Energy 87 (2010) 298-309.

[3] R. Khan, Small hydro power in india: is it sustainable business?, Appl Energy 152 (2015) 207-216.

[4] G. Bekele, G. Tadesse, Feasibility study of small Hydro/PV/Wind hybrid system for off-grid rural electrification in Ethiopia, Appl Energy 97 (2012) 5-15.

[5] G.C. Bakos, Feasibility study of a hybrid wind/hydropower-system for low-cost electricity production, Appl Energy 72 (2002) 599-608.

[6] P. Zakkour, R. Gochin, J. Lester, Developing a sustainable energy strategy for a water utility. Part II: a review of potential technologies and approaches, Environ Manage 66 (2002) 115-125.

[7] A. Carravetta, G. Del Giudice, O. Fecarotta, H. Ramos, Energy production in water distribution networks: A PAT design strategy, Water Resour Manage 26 (2012) 3947-3959.

[8] M. Giugni, N. Fontana, A. Ranucci, Optimal location of PRVs and turbines in water distribution systems, J Water Resour Manage 140 (9) (2014) 06014004.

[9] V. Puleo, M.C. Fontanazza, V. Notaro, M. De Marchis, G. Freni, G. La Loggia, Pumps as turbines (PATs) in water distribution networks affected by intermittent service, J Hydroinf 16 (2) (2014) 259-271.

[10] A. Carravetta, O. Fecarotta, M. Sinagra, T. Tucciarelli, Cost-benefit analysis for hydropower production in water distribution networks by a pump as turbine, J Water Resour Plann Manage 140 (6) (2014) 04014002.

[11] R. Sitzenfrei, J. Von Leon, W. Rauch, Design and optimization of small hydropowe systems in water distribution networks based on 10-years simulation with Epanet2, Procedia Eng 89 (2014) 533-539.

[12] M. Rossi, M. Righetti, M. Renzi, Pump-as-Turbine for energy recovery applications: the case study of an aqueduct, Energy Procedia 101 (2016) 1207-1214.

[13] B. Capelo, M. Perez-Sanchez, J.F.P. Fernandes, H.M. Ramos, P.A. Lopez-Jimenez, P.J. Costa Branco, Electrical behavior of the pump working as turbine in off grid operation, Appl Energy 208 (2017) 302-311.

[14] G.M. Lima, Junior E. Luvizotto, B.M. Brentan, Selection and location of Pumps as Turbines substituting pressure reducing valves, Renew Energy 109 (2017) 392-405.

[15] M. Kramer, K. Terheiden, S. Wieprecht, Pumps as turbines for efficient energy recovery in water supply networks, Renew Energy 122 (2018) 17-25.

[16] J. Gallagher, I.M. Harris, A.J. Packwood, A. McNabola, A.P. Williams, A strategic assessment of micro-hydropower in the UK and Irish water industry: Identifying technical and economic constraints, Renew Energy 81 (2015) 808-815. 
[17] J. Gallagher, D. Styles, A. McNabola, A.P. Williams, Making green technology greener: Achieving a balance between carbon and resource savings through eco-design in hydropower systems, Resour Conserv Recycl 105 (2015) 11-17.

[18] J. Gallagher, D. Styles, A. McNabola, A.P. Williams, Life cycle environmental balance and greenhouse gas mitigation potential of micro-hydropower energy recovery in the water industry, J Cleaner Prod 99 (2015) 152-159.

[19] P.A. Su, B. Karney, Micro hydroelectric energy recovery in municipal water systems: A case study for Vancouver, Urban Water J 8 (2015) 678-690.

[20] P. Bansal, N. Marshall, Feasibility of hydraulic power recovery from waste energy in bio-gas scrubbing processes, Appl Energy 87 (2010) 1048-1053.

[21] M. Perez-Sanchez, F.J. Sanchez-Romero, P.A. Lopez-Jimenez, H.M. Ramos, PATs selection towards sustainability in irrigation networks: Simulated annealing as a water management tool, Renew Energy 116 (2018) 234-249.

[22] J.G. Morillo, A. McNabola, E. Camachoa, P. Montesinosa, J.A. Rodríguez Díaz, Hydro-power energy recovery in pressurized irrigation networks: A case study of an Irrigation District in the South of Spain, Agric Water Manag 204 (2018) 17-27.

[23] V. Sammartano, C. Arico, A. Carravetta, O. Fecarotta, T. Tucciarelli, Banki-michell optimal design by computational fluid dynamics testing and hydrodynamic analysis, Energies 6 (2013) 2362-2385.

[24] Carravetta, Armando, Derakhshan Houreh, Shahram, Ramos, Helena M. Pumps as Turbines: Fundamentals and Applications. Springer International Publishing 2018, Cham, Switzerland.

[25] H. Ramos, A. Borga, Pump as turbine: an unconventional solution to energy production, Urban Water 1 (1999) 261-263.

[26] H. Nautiyal, V.A. Kumar, Reverse running pumps analytical, experimental and computational study: A review, Renew Sustain Energy Rev 14 (2010) 2059-2067.

[27] S.V. Jain, R.N. Patel, Investigations on pump running in turbine mode: A review of the state-of-the-art, Renew Sustain Energy Rev 30 (2014) 841-868.

[28] M. Venturini, S. Alvisi, S. Simani, L. Manservigi, Energy production by means of pumps as turbines in water distribution networks, Energies 10 (2017) 1666, https: //doi.org/10.3390/en10101666.

[29] M. Binama, W.T. Su, X.B. Li, F.C. Li, X.Z.Shi. Wei, An, Investigation on pump as turbine (PAT) technical aspects for micro hydropower schemes: A state-of-the-art review, Renew Sustain Energy Rev 79 (2017) 148-179.

[30] W. Gao, X. Feng, The power target of a fluid machinery network in a circulating water system, Appl Energy 205 (2017) 847-854.

[31] J. Du, H. Yang, Z. Shen, J. Chen, Micro hydro power generation from water supply system in high rise buildings using pump as turbines, Energy 137 (2017) 431-440.

[32] Novara D, Carravetta A, Derakhshan S, McNabola A, Ramos HM. A Cost model for Pumps as Turbines and a comparison of design strategies for their use as energy recovery devices in Water Supply Systems. Energy Efficiency in Motor-Driven Systems EEMODS'17 - Rome; 6-8 September; 2017(b).

[33] Novara D, Derakhshan S, McNabola A, Ramos HM. Estimation of unit cost and maximum efficiency for Pumps as Turbines. In: 9th Eastern European IWA Young Water Professionals conference - Budapest; 24-27 May; 2017.

[34] M. De Marchis, B. Milici, R. Volpe, A. Messineo, Energy saving in water distribution network through pump as turbine generators: economic and environmental analysis, Energies 9 (2016) 877.

[35] Simani S, Alvisi S, Venturini M. Data-Driven Design of a Fault Tolerant Fuzzy Controller for a Simulated Hydroelectric System. In: Proc 9th IFAC Symposium on Fault Detection, Supervision and Safety for Technical Processes, September 2-4, 2015. Paris, France. 1090-5.

[36] Finotti S, Simani S, Alvisi S, Venturini M. Benchmarking of Advanced Control Strategies for a Simulated Hydroelectric System. In: Proc 13th European Workshop on Advanced Control and Diagnosis, ACD 2016, 17-18 November 2016, Lille, France, Journal of Physics: Conference Series, 783(1), 012041.

[37] S. Simani, S. Alvisi, M. Venturini, Fault tolerant control of a simulated hydroelectric system, Control Eng Pract 51 (2016) 13-25.

[38] Simani S, Alvisi S, Venturini M. Fault Tolerant Model Predictive Control Applied to a Simulated Hydroelectric System. In: Proceedings 3rd International Conference on Control and Fault-Tolerant Systems - SysTol'16, Barcelona, Spain, p. 251-6. IEEE Control Systems Society.
[39] M. De Marchis, C.M. Fontanazza, G. Freni, A. Messineo, B. Milici, E. Napoli, et al. Energy recovery in water distribution networks. Implementation of pumps as turbine in a dynamic numerical model, Procedia Eng 70 (2014) 439-448.

[40] R. Tao, R. Xiao, F. Wang, W. Liu, Cavitation behavior study in the pump mode of a reversible pump-turbine, Renew Energy 125 (2018) 655-667.

[41] S. Abazariyan, R. Rafee, S. Derakhshan, Experimental study of viscosity effects on a pump as turbine performance, Renew Energy 127 (2018) 539-547.

[42] Y. Hao, L. Tan, Symmetrical and unsymmetrical tip clearances on cavitation performance and radial force of a mixed flow pump as turbine at pump mode, Renew Energy 127 (2018) 368-376.

[43] J. Li, Y. Zhang, K. Liu, H. Xian, J. Yu, Numerical simulation of hydraulic force on the impeller of reversible pump turbines in generating mode, J Hydrodyn 29 (4) (2017) 603-609.

[44] T. Wang, C. Wang, F. Kong, Q. Gou, S. Yang, Theoretical, experimental, and numerical study of special impeller used in turbine mode of centrifugal pump as turbine, Energy 130 (2017) 473-485.

[45] S. Derakhshan, A. Nourbakhsh, Experimental study of characteristic curves of centrifugal pumps working as turbines in different specific speeds, Exp Therm Fluid Sci 32 (2008) 800-807.

[46] S. Derakhshan, A. Nourbakhsh, Theoretical, numerical and experimental investigation of centrifugal pumps in reverse operation, Exp Therm Fluid Sci 32 (2008) $1620-1627$.

[47] S. Yang, S. Derakhshan, F. Kong, Theoretical, Numerical and Experimental Prediction of Pump As Turbine Performance, Renew Energy 48 (2012) 507-513.

[48] S. Derakhshan, N. Kasaeian, Optimization, numerical, and experimental study of a propeller pump as turbine, Energy Resour Technol 136 (1) (2014) 012005.

[49] O. Fecarotta, A. Carravetta, H.M. Ramos, R. Martino, An improved affinity model to enhance variable operating strategy for pumps used as turbines, J Hydraul Res 54 (3) (2016) 332-341.

[50] S. Huang, G. Qiu, X. Su, J. Chen, W. Zou, Performance prediction of a centrifugal pump as turbine using rotor-volute matching principle, Renew Energy 108 (2018) 64-71.

[51] F. Pugliese, F. De Paola, N. Fontana, M. Giugni, G. Marini, Experimental characterization of two Pumps As Turbines for hydropower generation, Renew Energy 99 (2016) 180-187

[52] D. Buono, E. Frosina, A. Mazzone, U. Cesaro, A. Senatore, Study of a pump as turbine for a hydraulic urban network using a tridimensional CFD modeling methodology, Energy Procedia 82 (2015) 201-208.

[53] De Rose V, Martelli F, Milani M, Montorsi L. Numerical Analysis of Centrifugal Pumps Running in Turbine Mode Under Dynamic Operating Conditions. In: Proceedings of the ASME 2017 Power and Energy Conference, PowerEnergy 2017, June 26-30, 2017, Charlotte, North Carolina, USA. Paper No. ES2017-3372, p. V001T07A002

[54] S. Barbarelli, M. Amelio, G. Florio, Predictive model estimating the performances of centrifugal pumps used as turbines, Energy 107 (2016) 103-121.

[55] X. Tan, A. Engeda, Performance of centrifugal pumps running in reverse as turbine: Part II - systematic specific speed and specific diameter based performance prediction, Renew Energy 99 (2016) 188-197.

[56] S. Barbarelli, M. Amelio, G. Florio, Experimental activity at test rig validating correlations to select pumps running as turbines in microhydro plants, Energy Convers Manage 149 (2017) 781-797.

[57] M. Rossi, M. Renzi, A general methodology for performance prediction of pumps-as-turbines using Artificial Neural Networks, Renew Energy 128 (2018) 265-274.

[58] J.F. Gulich, Centrifugal pumps, 3rd ed., Springer, Berlin Heidelberg, 2010.

[59] Venturini, M., Alvisi, S., Simani, S., Manservigi, L., 2017, Comparison of Different Approaches to Predict the Performance of Pumps as Turbines (PATs). Proc. ECOS 2017, July 2 - 6 2017, San Diego, CA. Paper \#18.

[60] A. Carravetta, O. Fecarotta, G. Del Giudice, H. Ramos, Energy recovery in wate systems by PATs: a comparison among the different installation schemes, Procedia Eng 70 (2014) 275-284.

[61] Optimization Toolbox ${ }^{\mathrm{TM}}$ User's Guide. The MathWorks, Inc. 3 Apple Hill Drive, Natick, MA 1760-2098. September 2016. 